\title{
Tumor Findings Sponsor Defined Identifier
}

National Cancer Institute

\section{Source}

National Cancer Institute. Tumor Findings Sponsor Defined Identifier. NCI Thesaurus.

Code C119941.

One or more sponsor defined characters used to identify, name, or characterize the tumor findings assessment. 